\title{
Femtosecond response of polyatomic molecules to ultra-intense hard X-rays
}

\author{
A. Rudenko ${ }^{1}$, L. Inhester ${ }^{2,3}$, K. Hanasaki ${ }^{2,3,4}$, X. Li ${ }^{1}$, S. J. Robatjazi ${ }^{1}$, B. $\operatorname{Erk}^{5}$, R. Boll ${ }^{5,6}$, K. Toyota ${ }^{2,3}$, Y. Hao $^{2,3,7}$, O. Vendrell $^{2,3,8}$, \\ C. Bomme ${ }^{5}$, E. Savelyev ${ }^{5}$, B. Rudek ${ }^{9}$, L. Foucar ${ }^{10}$, S. H. Southworth ${ }^{11}$, C. S. Lehmann ${ }^{11,12}$, B. Kraessig ${ }^{11}$, T. Marchenko ${ }^{13}$, \\ M. Simon ${ }^{13}$, K. Ueda ${ }^{14}$, K. R. Ferguson ${ }^{15}$, M. Bucher ${ }^{11,15}$, T. Gorkhover ${ }^{15,16,17}$, S. Carron ${ }^{15}$, R. Alonso-Mori ${ }^{15}$, J. E. Koglin ${ }^{15}$, \\ J. Correa ${ }^{2,5}$, G. J. Williams ${ }^{15,18}$, S. Boutet ${ }^{15}$, L. Young ${ }^{11,19}$, C. Bostedt ${ }^{11,20}$, S.-K. Son ${ }^{2,3}$, R. Santra ${ }^{2,3,21}$ \& D. Rolles ${ }^{1,5}$
}

$\mathrm{X}$-ray free-electron lasers enable the investigation of the structure and dynamics of diverse systems, including atoms, molecules, nanocrystals and single bioparticles, under extreme conditions ${ }^{1-7}$. Many imaging applications that target biological systems and complex materials use hard $\mathrm{X}$-ray pulses with extremely high peak intensities (exceeding $10^{20}$ watts per square centimetre) ${ }^{3,5}$. However, fundamental investigations have focused mainly on the individual response of atoms and small molecules using soft $\mathrm{X}$-rays with much lower intensities ${ }^{8-17}$. Studies with intense $X$-ray pulses have shown that irradiated atoms reach a very high degree of ionization, owing to multiphoton absorption ${ }^{8,12,13,18}$, which in a heteronuclear molecular system occurs predominantly locally on a heavy atom (provided that the absorption cross-section of the heavy atom is considerably larger than those of its neighbours) and is followed by efficient redistribution of the induced charge ${ }^{14-17,19,20}$. In serial femtosecond crystallography of biological objects-an application of X-ray free-electron lasers that greatly enhances our ability to determine protein structure $e^{2,3}$ - the ionization of heavy atoms increases the local radiation damage that is seen in the diffraction patterns of these objects ${ }^{21,22}$ and has been suggested as a way of phasing the diffraction data ${ }^{23,24}$. On the basis of experiments using either soft or less-intense hard X-rays ${ }^{14-19,25}$, it is thought that the induced charge and associated radiation damage of atoms in polyatomic molecules can be inferred from the charge that is induced in an isolated atom under otherwise comparable irradiation conditions. Here we show that the femtosecond response of small polyatomic molecules that contain one heavy atom to ultra-intense (with intensities approaching $10^{20}$ watts per square centimetre), hard (with photon energies of 8.3 kiloelectronvolts) $\mathrm{X}$-ray pulses is qualitatively different: our experimental and modelling results establish that, under these conditions, the ionization of a molecule is considerably enhanced compared to that of an individual heavy atom with the same absorption cross-section. This enhancement is driven by ultrafast charge transfer within the molecule, which refills the core holes that are created in the heavy atom, providing further targets for inner-shell ionization and resulting in the emission of more than 50 electrons during the $\mathrm{X}$-ray pulse. Our results demonstrate that efficient modelling of $\mathrm{X}$-ray-driven processes in complex systems at ultrahigh intensities is feasible.

In our experiments (see Methods for details), we exposed isolated xenon atoms and gas-phase iodomethane $\left(\mathrm{CH}_{3} \mathrm{I}\right)$ and iodobenzene $\left(\mathrm{C}_{6} \mathrm{H}_{5} \mathrm{I}\right)$ molecules to pulses generated at the Linac Coherent
Light Source (LCLS) with photon energies of $8.3 \mathrm{keV}$ and intensities exceeding $10^{19} \mathrm{~W} \mathrm{~cm}^{-2}$, and measured the yields and kinetic energies of the ionic fragments that were created. The accompanying firstprinciples theoretical study uses the XMOLECULE toolkit ${ }^{26}$, extended to include $a b$ initio photoionization cross-sections and decay rates ${ }^{27}$, to calculate the same set of observables for the case of iodomethane under the given experimental conditions, and to predict the time-dependent evolution of fragment charges and the molecular geometry.

Figure 1a displays the measured distributions of the charge state of iodine ions that were emitted from $\mathrm{CH}_{3} \mathrm{I}$ and $\mathrm{C}_{6} \mathrm{H}_{5} \mathrm{I}$ molecules at the maximum available pulse energy and the results for xenon atoms under the same experimental conditions. Each X-ray pulse had a duration of less than $30 \mathrm{fs}$ (full-width at half-maximum, FWHM) and an average pulse shape as shown in Extended Data Fig. 1. Xenon and iodine have a very similar absorption cross-sections at $8.3 \mathrm{keV}$ $\left(0.056 \mathrm{Mb}\right.$ and $0.052 \mathrm{Mb}$, respectively $\left.{ }^{28} ; 1 \mathrm{Mb}=10^{-18} \mathrm{~cm}^{2}\right)$. Figure $1 \mathrm{a}$ demonstrates that xenon atoms can reach a very high level of ionization $(48+)$, which is limited by the last ionic state that can be ionized by one $8.3-\mathrm{keV}$ photon ${ }^{28}$. Almost the same charge state $(47+)$ can be observed for the iodine ions from $\mathrm{CH}_{3} \mathrm{I}$ molecules. This similarity is in contrast to previous observations in the soft X-ray domain ${ }^{14-17}$ and to results at $5.5 \mathrm{keV}$ (refs 18,19), from which the measured charge of an isolated heavy atom was found to be considerably larger than for a similar atom embedded in a molecule. The highest charge state of carbon we observe in this experiment is $\mathrm{C}^{4+}$ (see Extended Data Fig. 2). The absence of higher charge states of carbon is due to the fact that (multiphoton) absorption by the carbon atom is negligible because of its low cross-section at this photon energy, such that the high charge states stem exclusively from charge transfer ${ }^{16,17}$ Applying a semi-classical over-the-barrier charge-transfer model, which has previously been discussed in the context of multiphoton ionization of $\mathrm{CH}_{3} \mathrm{I}$ in the soft X-ray domain ${ }^{16,17}$, we find that only the four valence electrons can be removed from the carbon atom.

To determine the total charge of the $\mathrm{CH}_{3}$ I molecule, we present in Fig. $1 \mathrm{~b}$ the yield of iodine and carbon ions that were detected in coincidence for an experiment with slightly reduced intensity. It can clearly be seen from Fig. $1 b$ that the highest iodine charge states (starting from $\mathrm{I}^{20+}$ ) are always detected in coincidence with $\mathrm{C}^{4+}$ ions. Because a hydrogen atom cannot remain neutral in the vicinity of highly charged iodine and carbon ions, we deduce that the maximal total charge of the molecule amounts to $54+\left(\mathrm{I}^{47+}+\mathrm{C}^{4+}+3 \mathrm{H}^{+}\right)$, which considerably exceeds the highest charge state that we observed for an isolated xenon atom $(48+)$.

\footnotetext{
${ }^{1}$ J. R. Macdonald Laboratory, Department of Physics, Kansas State University, Manhattan, Kansas, USA. ${ }^{2}$ Center for Free-Electron Laser Science, Deutsches Elektronen-Synchrotron (DESY), Hamburg, Germany. ${ }^{3}$ The Hamburg Centre for Ultrafast Imaging, Hamburg, Germany. ${ }^{4}$ Department of Chemistry, Graduate School of Science, Tohoku University, Sendai, Japan. ${ }^{5}$ Deutsches Elektronen-Synchrotron (DESY), Hamburg, Germany. ${ }^{6}$ Max Planck Institute for Nuclear Physics, Heidelberg, Germany. ${ }^{7}$ Department of Physics, University of Science and Technology Beijing, Beijing, China. ${ }^{8}$ Department of Physics and Astronomy, Aarhus University, Aarhus, Denmark. ${ }^{9}$ Physikalisch-Technische Bundesanstalt (PTB), Braunschweig, Germany. ${ }^{10}$ Max Planck Institute for Medical Research, Heidelberg, Germany. ${ }^{11}$ Argonne National Laboratory, Lemont, Illinois, USA. ${ }^{12}$ Faculty of Chemistry, Philipps-Universität Marburg, Marburg, Germany. ${ }^{13}$ Sorbonne Universités, UPMC Université Paris 06, CNRS, LCP-MR (UMR 7614), Paris, France. ${ }^{14}$ Institute of Multidisciplinary Research for Advanced Materials, Tohoku University, Sendai, Japan. ${ }^{15}$ Linac Coherent Light Source (LCLS), SLAC National Accelerator Laboratory, Menlo Park, California, USA. ${ }^{16}$ PULSE Institute, SLAC, Menlo Park, California,USA. ${ }^{17}$ Institut für Optik und Atomare Physik, Technische Universität Berlin, Berlin,Germany. ${ }^{18}$ NSLS-II, Brookhaven National Laboratory, Upton, New York, USA. ${ }^{19}$ Department of Physics, University of Chicago, Chicago, Illinois, USA. ${ }^{20}$ Department of Physics and Astronomy, Northwestern University, Evanston, Illinois, USA. ${ }^{21}$ Department of Physics, University of Hamburg, Hamburg, Germany.
} 


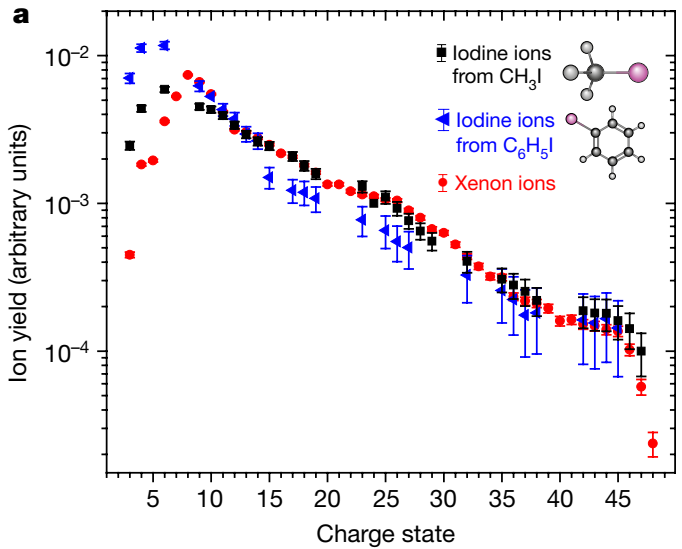

Figure 1 | Experimental charge-state distributions. a, Charge-state distributions of iodine ions emitted from $\mathrm{CH}_{3} \mathrm{I}$ (black squares) and $\mathrm{C}_{6} \mathrm{H}_{5} \mathrm{I}$ (blue triangles) molecules upon irradiation by $8.3-\mathrm{keV}$ pulses with an average pulse energy of $1.1 \mathrm{~mJ}$. Red circles show the charge-state distribution obtained from atomic xenon under the same conditions. The data are accumulated over 287,400 shots for $\mathrm{CH}_{3} \mathrm{I}, 212,880$ shots for $\mathrm{C}_{6} \mathrm{H}_{5} \mathrm{I}$ and 372,600 shots for xenon. The curves are normalized to have an integral

To shed light on the mechanism of this enhanced molecular ionization, we present in Fig. 2a a comparison of the results of a theoretical analysis of the multiple X-ray ionization of $\mathrm{CH}_{3} \mathrm{I}$ using the XMOLECULE toolkit ${ }^{26}$. Figure 2 a displays the calculated charge-state
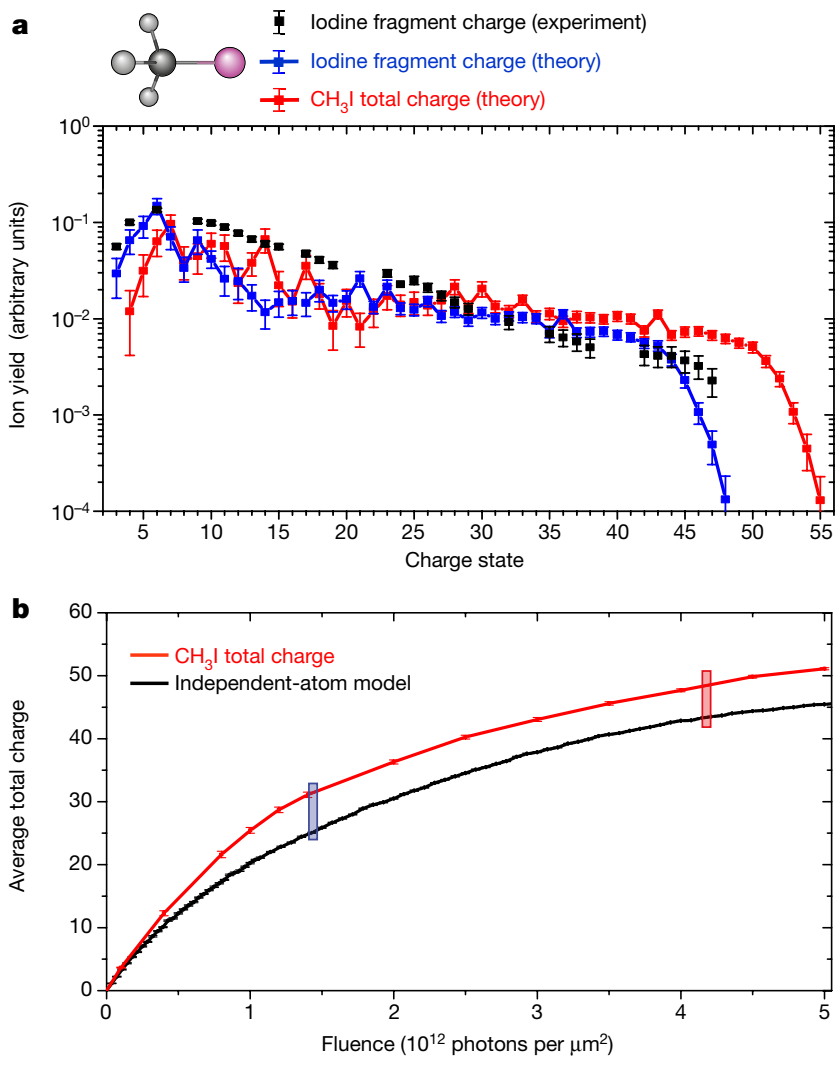

c
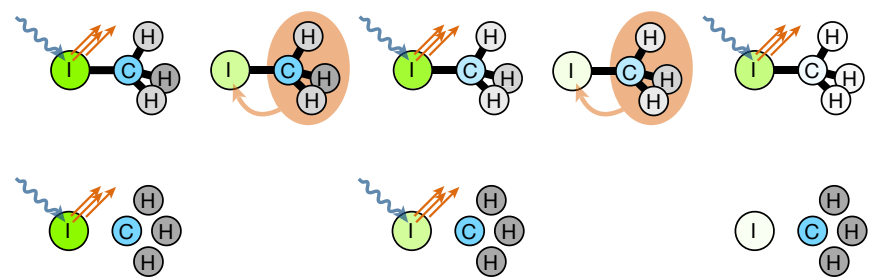

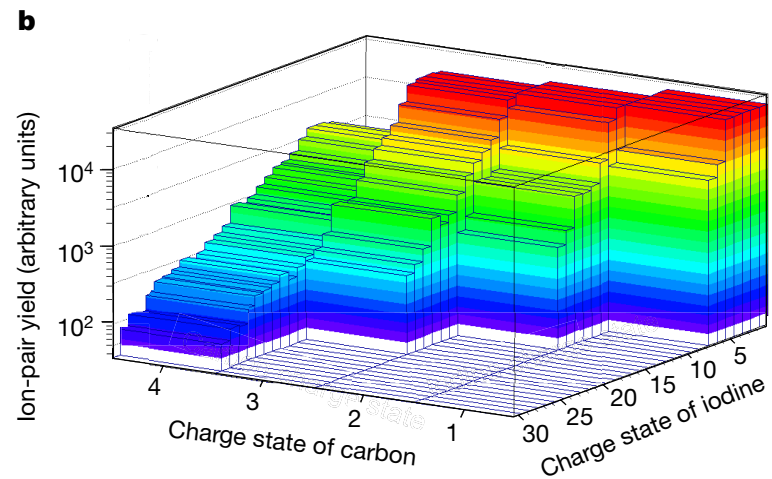

charge of 1 . The error bars reflect the uncertainty of the data (1 s.d.) due to the finite counting statistics. The $\mathrm{CH}_{3} \mathrm{I}$ and $\mathrm{C}_{6} \mathrm{H}_{5} \mathrm{I}$ molecules are sketched above the plot, with the iodine atom highlighted in purple, carbon atoms in grey and hydrogen atoms in white. $\mathbf{b}$, Yield of carbon and iodine ion pairs detected in coincidence after $\mathrm{CH}_{3} \mathrm{I}$ ionization by pulses with the same parameters as in a but with the pulse energy reduced to $0.4 \mathrm{~mJ}$.

The data are accumulated over $1,242,850$ shots.

distribution of iodine ions and the total charge of the molecule. The results of the calculation are averaged over the spatial distribution of the pulse intensity so that they can be compared to the experimental data for iodine ions. The theoretical predictions agree reasonably well with the outcome of the experiment, indicating that our theoretical model adequately describes the essential mechanisms of $\mathrm{CH}_{3} \mathrm{I}$ ionization in ultra-intense hard X-ray pulses. The calculations also show that the total charge of the molecule for high charge states is shifted by 7 with respect to the charge-state distribution for iodine, in good agreement with the experimental finding that $\mathrm{C}^{4+}$ and $3 \mathrm{H}^{+}$ions are produced when the molecule is highly ionized. To understand the fluence dependence of the molecular ionization process, we present in Fig. $2 \mathrm{~b}$ the average total charge state of the $\mathrm{CH}_{3} \mathrm{I}$ molecule calculated as a function of the fluence (without focal volume averaging) and the prediction of an independent-atom model. The results show that for low fluences (at which earlier experiments ${ }^{14-19,25}$ have been conducted) the total charge state is equivalent to the case of a system of isolated atoms (which, in turn, is nearly identical to that of the heavy atom), whereas molecular effects considerably enhance the level of ionization at higher fluences.

This enhancement can be qualitatively understood by recurrent charge redistribution upon multiphoton X-ray absorption, as sketched in Fig. 2c. At $8.3 \mathrm{keV}$, photoabsorption occurs predominantly in the $2 s$ and $2 p$ shells of iodine, and the subsequent Auger decay of these L-shell vacancies is followed by a cascade of further Auger processes that creates a high charge that is initially localized at the iodine site.

Figure 2 | Enhanced ionization of the molecule. a, Calculated (blue) and measured (black) charge-state distribution of iodine ions from $\mathrm{CH}_{3} \mathrm{I}$ molecules. The pulse parameters are the same as for Fig. 1a. The experimental data are accumulated over 287,400 shots. The calculated total charge of the molecule is shown in red. The error bars reflect the statistical uncertainty of the data ( 1 s.d.). The $\mathrm{CH}_{3} \mathrm{I}$ molecule is sketched above the plot, with atoms coloured as in Fig. 1a. b, Average total molecular charge as a function of fluence calculated for $\mathrm{CH}_{3} \mathrm{I}$ molecules (red) and within the independent-atom model (black). The fluence values corresponding to Fig. $1 \mathrm{a}$ and $\mathrm{b}$ are marked with red and blue bars, respectively. The error bars show the standard error of the mean. c, Illustration of the CREXIM mechanism. In the molecule (upper row), the repeated ionization (blue and orange arrows) of the iodine atom drives electrons from the methyl group to the iodine (orange shading), such that there are more electrons available for ionization compared to independent atoms (lower row). The darkness of the shading of the atoms indicates the number of electrons that remain in the atoms. 
Direct photoabsorption by the carbon or hydrogen atoms is negligible, owing to very small cross-sections. The created charge imbalance drives a rearrangement of electrons from the methyl group towards the iodine atom, refilling the created holes and thus lowering the charge of the latter. For each ionization step, this charge rearrangement occurs on a subfemtosecond timescale and so is much faster than the overall ionization dynamics, such that the electrons can be considered to rearrange instantaneously. For single-photon absorption, the total molecular charge remains essentially the same after this rearrangement, with only a slight enhancement due to the new relaxation channels that are possible in a molecular environment ${ }^{29}$. In the high-fluence regime, however, other X-ray photons are likely to be absorbed by the heavy atom, cycling through this charge redistribution process multiple times. At the peak intensities reached in our experiment, the iodine fragment sequentially absorbs more than $20 \mathrm{X}$-ray photons, and the total charge of the molecule is mainly limited by the number of electrons that are available from the molecular environment to fill the vacancies that are created at the iodine site.

We predict that this charge-rearrangement-enhanced X-ray ionization of molecules (CREXIM), which we report here for $\mathrm{CH}_{3} \mathrm{I}$, plays an important part in the quantitative understanding of radiation damage of polyatomic systems irradiated by very intense X-ray pulses, and will be even more pronounced for larger systems. A first indication is given by our experimental results for iodobenzene molecules shown in Fig. 1a, for which we observe the charging of a heavy fragment to a degree comparable to the case of isolated atoms despite the presence of a benzene ring, which can provide numerous additional electrons as compared to the case of $\mathrm{CH}_{3} \mathrm{I}$. This is very different from the outcome of previous experiments with iodouracil molecules under less intense hard X-rays ${ }^{25}$, in which the highest iodine charge state detected from the molecule was $\mathrm{I}^{4+}$, compared to $\mathrm{Xe}^{18+}$ that was produced under similar experimental conditions from isolated xenon atoms ${ }^{18}$. Even though we cannot unambiguously determine the total molecular charge for $\mathrm{C}_{6} \mathrm{H}_{5} \mathrm{I}$, the measured distribution of light ionic fragments, which is dominated by $\mathrm{CH}^{+}, \mathrm{C}^{+}, \mathrm{C}^{2+}, \mathrm{C}^{3+}$ and $\mathrm{H}^{+}$ions, indicates that, on average, they carry more than 10 charges in total. Therefore, we conclude that for the highest intensities, for which the iodine charge state reaches $\mathrm{I}^{45+}$, the total charge of $\mathrm{C}_{6} \mathrm{H}_{5} \mathrm{I}$ exceeds the value of $54+$ reached for $\mathrm{CH}_{3} \mathrm{I}$.

For a small molecular system such as $\mathrm{CH}_{3} \mathrm{I}$, the theoretical model described here enables detailed analysis of the evolving electronic structure that is interweaved with nuclear dynamics. To further test

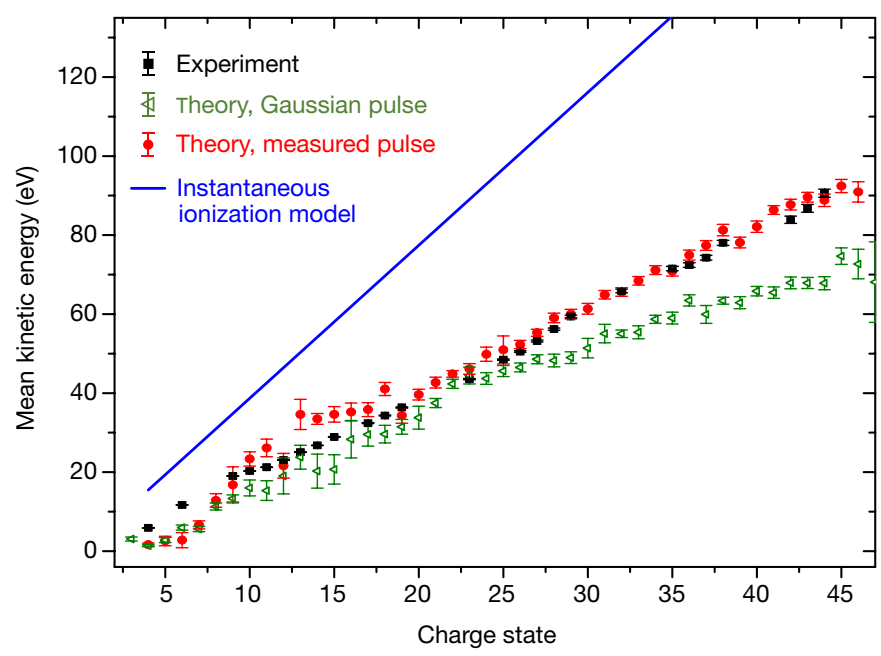

Figure 3 | Kinetic energies of the iodine ions. Measured (black) and calculated (red, green) mean values of the kinetic-energy distribution (KED) of iodine ions from $\mathrm{CH}_{3} \mathrm{I}$ are shown as a function of their charge state. The pulse parameters are the same as for Fig. 1a. The experimental data are accumulated over 287,400 shots. For the calculation, green triangles represent the results obtained using a Gaussian pulse shape with a FWHM of $30 \mathrm{fs}$, whereas red circles depict the values obtained using the measured pulse profile. The error bars show the standard error of the mean. The blue line depicts the energies that are expected for instantaneous ionization.

the predictive power of the model, we compare the measured and calculated kinetic energies for iodine ions in Fig. 3. The kinetic energies of the fragments are sensitive to the detailed fragmentation dynamics and, in particular, set boundary conditions for the times and internuclear separations at which the ionic charges are formed. The kinetic energies that are expected for an instantaneous ionization to a given final charge state are also plotted, which substantially overestimate the measured values because the charging does not happen instantaneously. Our analysis shows that the kinetic-energy distribution (KED) is sensitive to the temporal pulse shape used for the calculations, so the experimentally determined temporal profile of the X-ray pulse ${ }^{30}$ (see Methods and Extended Data Fig. 1) needs to be taken into account. As can be seen from Fig. 3, calculations for Gaussian pulses do not match the experimental values, especially for the highest charge states. In contrast,

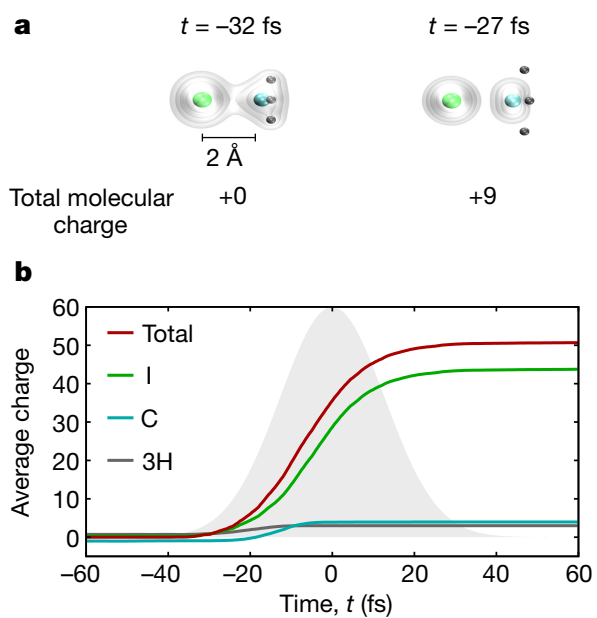

Figure 4 | Simulated evolution of molecular geometry and charge distribution. a, Snapshots of molecular charges and nuclear positions at different times $t$ before and after the peak of the X-ray pulse $(t=0)$ for an exemplary trajectory. The atoms are coloured as in Fig. 2c. Contour lines of the electron density are shown as shaded grey areas. The internuclear
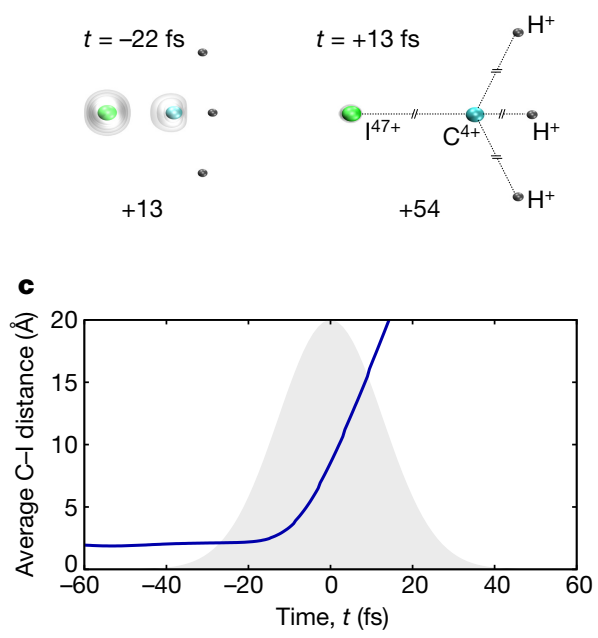

separations in the right-most panel are not to scale. $\mathbf{b}$, Time-dependent average partial charge of different fragments and total charge. c, Timedependent average carbon-iodine distance. The shaded grey areas in $\mathbf{b}$ and c depict the temporal envelope of a Gaussian pulse with a 30-fs FWHM. A fluence of $5 \times 10^{12}$ photons per $\mu \mathrm{m}^{2}$ is used. 
the mean values of the calculated KEDs that were obtained using the measured pulse profile agree well with the experimental values.

The above results demonstrate that our first-principles theoretical model can quantitatively describe the main trends in the fragmentation of a small polyatomic molecule exposed to an ultra-intense hard X-ray pulse. This model can be used to predict the time-dependent evolution of the charge distribution directly and the molecular geometry in a fragmenting molecule, as illustrated in Fig. 4. Figure 4a displays a few snapshots of such a 'molecular movie', revealing the way in which the $\mathrm{CH}_{3} \mathrm{I}$ molecule is charged up to $54+$. With a larger pool of electrons available for charge rearrangement, we expect a stronger CREXIM effect, unless the atoms involved in the charge rearrangement are quickly separated by dissociation. Figure $4 \mathrm{~b}, \mathrm{c}$ demonstrates that the final charge states for all four light fragments are reached at relatively small C-I distances well before the maximum of the X-ray pulse. This suggests that CREXIM is not much affected by nuclear motions in this case, which is consistent with a weak dependence of the calculated total molecular charge on the nuclear dynamics (see Extended Data Fig. 3). This result further supports our prediction that for larger polyatomic systems the CREXIM effect plays an even more important part than for $\mathrm{CH}_{3} \mathrm{I}$.

The experimental and theoretical results presented here provide a detailed picture of the response of a small polyatomic molecule to a femtosecond hard X-ray pulse at peak intensities as high as $2 \times 10^{19} \mathrm{~W} \mathrm{~cm}^{-2}$. They clearly establish that the interaction of ultraintense, hard X-rays with molecules is fundamentally different from the interaction with isolated atoms, and can be understood and described only when accounting for molecular charge rearrangements that greatly enhance the degree of ionization and overall X-ray absorption when heavy elements are present. Even though more than 50 electrons can be emitted from a molecule within a few tens of femtoseconds, the ionization process can still be quantitatively described by an intuitive sequential model that involves a series of single-photon absorption steps. This opens up a way for efficient modelling of X-ray-driven processes in complex systems at ultra-high intensities, including a quantitative description of local radiation damage that occurs on the timescale of the X-ray pulse duration and will therefore need to be accounted for when interpreting coherent diffractive imaging data.

Online Content Methods, along with any additional Extended Data display items and Source Data, are available in the online version of the paper; references unique to these sections appear only in the online paper.

\section{Received 14 October 2016; accepted 3 April 2017.}

1. Marangos, J. P. Introduction to the new science with $x$-ray free electron lasers Contemp. Phys. 52, 551-569 (2011).

2. Chapman, H. N. et al. Femtosecond X-ray protein nanocrystallography. Nature 470, 73-77 (2011).

3. Schlichting, I. \& Miao, J. Emerging opportunities in structural biology with X-ray free electron lasers. Curr. Opin. Struct. Biol. 22, 613-626 (2012).

4. Vinko, S. M. et al. Creation and diagnostics of a solid-density plasma with an X-ray free-electron laser. Nature 482, 59-62 (2012).

5. Neutze, R. et al. Potential for biomolecular imaging with femtosecond X-ray pulses. Nature 406, 752-757 (2000).

6. Seibert, M. M. et al. Single mimivirus particles intercepted and imaged with an X-ray laser. Nature 470, 78-81 (2011).

7. Loh, N. D. et al. Fractal morphology, imaging and mass spectrometry of single aerosol particles in flight. Nature 486, 513-517 (2012).

8. Young, L. et al. Femtosecond electronic response of atoms to ultra-intense X-rays. Nature 466, 56-61 (2010).

9. Hoener, M. et al. Ultraintense X-ray induced ionization, dissociation, and frustrated absorption in molecular nitrogen. Phys. Rev. Lett. 104, 253002 (2010).

10. Fang, L. et al. Double core-hole production in $\mathrm{N}_{2}$ : beating the Auger clock. Phys. Rev. Lett. 105, 083005 (2010).

11. Doumy, G. et al. Nonlinear atomic response to intense ultrashort $X$ rays. Phys. Rev. Lett. 106, 083002 (2011).

12. Rudek, B. et al. Ultra-efficient ionization of heavy atoms by intense X-ray free-electron laser pulses. Nat. Photon. 6, 858-865 (2012).

13. Rudek, B. et al. Resonance-enhanced multiple ionization of krypton at an X-ray free-electron laser. Phys. Rev. A 87, 023413 (2013).
14. Erk, B. et al. Ultrafast charge rearrangement and nuclear dynamics upon inner-shell multiple ionization of small polyatomic molecules. Phys. Rev. Lett 110, 053003 (2013)

15. Erk, B. et al. Inner-shell multiple ionization of polyatomic molecules with an intense X-ray free-electron laser studied by coincident ion momentum imaging. J. Phys. At. Mol. Opt. Phys. 46, 164031 (2013).

16. Erk, B. et al. Imaging charge transfer in iodomethane upon X-ray photoabsorption. Science 345, 288-291 (2014)

17. Boll, R. et al. Charge transfer in dissociating iodomethane and fluoromethane molecules ionized by intense femtosecond X-ray pulses. Struct. Dyn. 3, 043207 (2016).

18. Fukuzawa, H. et al. Deep inner-shell multiphoton ionization by intense X-ray free-electron laser pulses. Phys. Rev. Lett. 110, 173005 (2013).

19. Motomura, K. et al. Charge transfer and nuclear dynamics following deep inner-shell multiphoton ionization of $\mathrm{CH}_{3}$ I molecules by X-ray free-electron laser pulses. J. Phys. Chem. Lett. 6, 2944-2949 (2015).

20. Stumpf, V., Gokhberg, K. \& Cederbaum, L. S. The role of metal ions in X-ray-induced photochemistry. Nat. Chem. 8, 237-241 (2016).

21. Nass, K. et al. Indications of radiation damage in ferredoxin microcrystals using high-intensity X-FEL beams. J. Synchrotron Radiat. 22, 225-238 (2015).

22. Galli, L. et al. Electronic damage in $\mathrm{S}$ atoms in a native protein crysta induced by an intense X-ray free-electron laser pulse. Struct. Dyn. 2, 041703 (2015).

23. Son, S.-K., Chapman, H. N. \& Santra, R. Multiwavelength anomalous diffraction at high X-ray intensity. Phys. Rev. Lett. 107, 218102 (2011).

24. Galli, L. et al. Towards phasing using high X-ray intensity. IUCrJ 2, 627-634 (2015)

25. Nagaya, K. et al. Ultrafast dynamics of a nucleobase analogue illuminated by a short intense X-ray free electron laser pulse. Phys. Rev. X 6, 021035 (2016).

26. Hao, Y., Inhester, L., Hanasaki, K., Son, S.-K. \& Santra, R. Efficient electronic structure calculation for molecular ionization dynamics at high x-ray intensity. Struct. Dyn. 2, 041707 (2015)

27. Inhester, L., Hanasaki, K., Hao, Y., Son, S.-K. \& Santra, R. X-ray multiphoton ionization dynamics of a water molecule irradiated by an $\mathrm{X}$-ray free-electron laser pulse. Phys. Rev. A 94, 023422 (2016).

28. Jurek, Z., Son, S.-K., Ziaja, B. \& Santra, R. XMDYN and XATOM: versatile simulation tools for quantitative modeling of $X$-ray free-electron laser induced dynamics of matter. J. Appl. Cryst. 49, 1048-1056 (2016).

29. Dunford, R. W. et al. Evidence for interatomic Coulombic decay in Xe K-shell-vacancy decay of $\mathrm{XeF}_{2}$. Phys. Rev. A 86, 033401 (2012).

30. Behrens, C. et al. Few-femtosecond time-resolved measurements of X-ray free-electron lasers. Nat. Commun. 5, 3762 (2014).

Acknowledgements This work is supported by the US Department of Energy, Office of Science, Basic Energy Sciences, Chemical Sciences, Geosciences, and Biosciences Division, who supported the Kansas group under contract no. DE-FG02-86ER13491 and the Argonne group under contract no. DE-AC02-06CH11357, and by the excellence cluster 'The Hamburg Centre for Ultrafast Imaging: Structure, Dynamics, and Control of Matter at the Atomic Scale' of the Deutsche Forschungsgemeinschaft. Use of the Linac Coherent Light Source (LCLS), SLAC National Accelerator Laboratory, is supported by the US Department of Energy, Office of Science, Office of Basic Energy Sciences under contract no. DE-AC02-76SF00515. A.R. acknowledges support from the National Science Foundation EPSCoR Track II Award No. IIA-1430493. T.G. acknowledges the Peter-Ewald Fellowship from the Volkswagen foundation. D.R. acknowledges support from the Helmholtz Gemeinschaft through the Helmholtz Young Investigator Program. K.U. acknowledges the XFEL strategy programme of MEXT, the five stars alliance and the TAGEN project for support. We are grateful to the SLAC staff for their support and hospitality during the beamtime.

Author Contributions A.R., B.R. and D.R. conceived the experiment, which was coordinated by A.R. and D.R. and carried out by A.R., B.E., R.B., C.Bom., E.S., B.R., L.F., S.H.S., C.S.L., B.K., T.M., M.S., K.U., K.R.F., M.B., T.G., S.C., R.A.-M., J.E.K., J.C., G.J.W., S.B., L.Y., C.Bos. and D.R. The ion spectrometer with the delay-line detector was assembled and operated mainly by A.R., B.E., R.B., C.Bom., E.S., B.R. and D.R. K.R.F., T.G., M.B. and C.Bos. were responsible for molecular beam alignment and operation. The LCLS CXI beamline and related LCLS equipment was mainly operated by R.A.-M., J.E.K., G.J.W. and S.B. Data acquisition was coordinated by L.F. and S.C. X.L. and S.J.R. analysed the data with help from B.E., R.B. and L.F. under guidance from A.R. and D.R. L.I., K.H., Y.H., O.V., S.-K.S. and R.S. developed the XMOLECULE toolkit and carried out the calculations. K.T. conducted the calibration of the pulse parameters with the atomic argon data. A.R., L.I., S.-K.S., R.S. and D.R. interpreted the results and wrote the manuscript with input from all authors.

Author Information Reprints and permissions information is available at www.nature.com/reprints. The authors declare no competing financial interests. Readers are welcome to comment on the online version of the paper. Publisher's note: Springer Nature remains neutral with regard to jurisdictional claims in published maps and institutional affiliations. Correspondence and requests for materials should be addressed to A.R. (rudenko@phys.ksu.edu) or S.-K.S. (sangkil.son@cfel.de). 


\section{METHODS}

Experimental set-up. The experiment was performed at the Coherent X-ray Imaging (CXI) beamline ${ }^{31}$ of the LCLS free-electron laser (FEL) facility at SLAC, operated at a $120-\mathrm{Hz}$ repetition rate with a photon energy of $8.3 \mathrm{keV}$. A sketch of the experimental set-up is shown in Extended Data Fig. 4. Using a pair of Kirkpatrick-Baez mirrors, the LCLS X-ray beam was focused in the centre of the CXI vacuum chamber, where it crossed a collimated pulsed beam of cold atoms or molecules. For the iodomethane and iodobenzene measurements, the sample reservoir containing the liquid sample was at room temperature, and helium at a backing pressure of 4 bar was used as the carrier gas. The ionic fragments produced by the interaction of the X-rays with the target gas were guided by a constant homogeneous electric field of $405 \mathrm{~V} \mathrm{~cm}^{-1}$ onto a time- and position-sensitive $80-\mathrm{mm}$ microchannel plate (MCP) detector equipped with a delay-line anode. The MCP and delay-line signal traces were recorded for each FEL shot with an Acqiris DC282 digitizer. Each ion hit was identified in the post-analysis using a software version of a constant-fraction discriminator such that the effect of pulse height variations due to varying MCP efficiency for different ionic charge states was negligible. Extended Data Fig. 2 displays the yield of ionic fragments from the break-up of the $\mathrm{CH}_{3} \mathrm{I}$ molecules, corresponding to the data in Fig. 1a, measured as a function of the ion time-of-flight (TOF) and hit position on the detector. From these TOFs and hit positions, the three-dimensional momentum vectors of the detected ions were calculated. All of the iodine ions created and all of the carbon ions up to a kinetic energy of $110 \mathrm{eV}$ were detected. As can be seen from Extended Data Fig. 2, the faster carbon ions can be lost if emitted at large angle towards the spectrometer axis.

At the highest intensity used in the experiment, coincident detection of ionic fragments was prevented by a large number of particles hitting the detector; therefore, for the coincidence measurement (Fig. 1b), the intensity had to be reduced to about $35 \%$ of its maximum value.

Because the X-ray pulse energies at LCLS are measured by gas detectors located upstream of the beamline optics, these values do not include the transmission losses in the beamline and so have to be corrected to calculate the actual X-ray fluence in the interaction region. For this purpose, we performed calibration measurements on argon atoms, as described below. All of the X-ray pulse energies quoted in the text are the pulse energies in the interaction region ('on target') that were calculated from the measured gas monitor detector values using a beamline transmission of $32 \%$ (determined from these calibration measurements).

Determining the $\mathrm{X}$-ray fluence, and spatial and temporal $\mathrm{X}$-ray beam profiles. To determine the X-ray fluence on target and the spatial beam profile in the experiment, which are necessary quantities for the theoretical modelling, the charge-state distribution of argon atoms was measured under the same experimental conditions as used for the measurements on xenon, $\mathrm{CH}_{3} \mathrm{I}$ and $\mathrm{C}_{6} \mathrm{H}_{5} \mathrm{I}$. The calibration procedure is similar to that described in the supplementary material of ref. 18. The fluence distribution function is fitted to provide good agreement between theoretical and experimental charge-state distributions of the argon atom. For a photon energy of $8.3 \mathrm{keV}$, we achieve the best fit to the experimental data on argon when assuming a beamline transmission of $32 \%$ and a double-Gaussian spatial distribution (similar to that determined previously ${ }^{32}$ ), with a focal spot size of $0.35 \mu \mathrm{m} \times 0.3 \mu \mathrm{m}$ and a ratio of peak fluences and widths of the two Gaussians of 0.16 and 2.5, respectively. In Extended Data Fig. 5 we show the experimental charge-state distributions for argon compared to the ion yields calculated using the above parameters. Extended Data Fig. 6 depicts the corresponding fluence distribution function, which is then used to calculate the charge-state distribution and the kinetic energies of the fragments that are compared with the experimental data for $\mathrm{CH}_{3} \mathrm{I}$ in Figs 2a and 3, respectively.

The temporal profile of the X-ray pulses at LCLS was measured shot by shot using the X-band transverse deflecting cavity (XTCAV; see ref. 30 for details). In Extended Data Fig. 1 we show the experimental pulse shape averaged over 369 $\mathrm{X}$-ray pulses for a nominal pulse duration of $30 \mathrm{fs}$ (FWHM), as measured in the experiment with the same X-ray beam parameters. The measured pulse shape has a distinct bimodal structure that differs substantially from a Gaussian-shaped pulse with a FWHM of $30 \mathrm{fs}$.

Theoretical modelling. The ionization and fragmentation dynamics of a $\mathrm{CH}_{3} \mathrm{I}$ molecule under irradiation with ultra-intense $\mathrm{X}$-rays are investigated using the newly developed toolkit, XMOLECULE ${ }^{26,27}$. To describe the multiphoton, multiple ionization dynamics, it solves a set of coupled rate equations with calculated cross-sections and rates ${ }^{27}$. In the course of multiphoton ionization at the given photon energy and fluence, all but the electrons in the K-shell of iodine can be ionized. The number of electronic configurations with 0,1 or 2 electrons in the relevant molecular orbitals in $\mathrm{CH}_{3} \mathrm{I}$ exceeds $2 \times 10^{14}$. To handle this large number of coupled rate equations for even a fixed molecular geometry, a kinetic Monte Carlo approach is imperative $e^{12,18}$. To describe nuclear motions, the nuclei are propagated classically during each kinetic Monte Carlo trajectory, with molecular forces, rates and cross-sections ${ }^{27}$ calculated on the fly from a given electronic configuration and molecular geometry.

The electronic structure is calculated on the basis of the Hartree-Fock-Slater model using numerical atomic basis functions. For each electron configuration and molecular geometry that is visited in a kinetic Monte Carlo trajectory, molecular orbitals are re-optimized to account for the adaptation of the electronic structure after each ionization step. Numerical basis functions consisting of a minimal set plus additional $6 s$ and $6 p$ basis functions on iodine are used. These basis functions are obtained using an atomic calculation conducted using $\mathrm{XATOM}^{28}$ and are adapted for the corresponding atomic core hole configurations. This procedure accounts for the effects of molecular charge rearrangement: molecular orbitals and basis functions are relaxed instantaneously for every respective electronic configuration such that, during sequential ionization steps, partial charges in the molecule may shift from one atom to another.

For all calculations, the initial geometry was taken from ref. 33 and initial velocities were assumed to be zero. The calculations were performed with a photon energy of $8.3 \mathrm{keV}$ and at 14 fluence points ranging from $1 \times 10^{11}$ photons per $\mu \mathrm{m}^{2}$ to $5 \times 10^{12}$ photons per $\mu \mathrm{m}^{2}$ and for various temporal pulse profiles. For each fluence point, about 200 Monte Carlo trajectories were sufficient to obtain converged charge-state distributions. To make a direct comparison with the LCLS experiment, we used the charge-state distribution for argon to determine the fluence distribution function of the X-ray beam, as described above.

To study the effect of nuclear dynamics on the total molecular charge, we also performed simulations with fixed nuclei. As can be seen from Extended Data Fig. 3, the average charge of the molecule shows only a weak dependence on nuclear dynamics, indicating that for such small systems the final charge state that is achieved is mainly determined by the electronic structure of the molecule, which defines the course of charge rearrangement in the early stages of the multiple ionization process.

Width of the measured and calculated KEDs of the ions. The experimental and calculated kinetic energies for each fragment ion have a rather broad distribution, which is only partially described by the mean kinetic energy shown in Fig. 3. To further test the limits of our modelling of the ionization and fragmentation dynamics, we compared the widths of the experimental and calculated KEDs, expressed in terms of standard deviations. In Extended Data Fig. 7 we show the mean and standard deviation of the measured kinetic energy releases compared with the calculated values. As can be seen, the variance of the calculated KED is much smaller than that of the measured KED. This discrepancy might originate from various effects that are disregarded in our modelling.

(1) There are some fluctuations in the X-ray pulse energy, which could cause fluctuations in the resulting kinetic energies of the fragments. However, calculations with different pulse energies show that fluctuations in pulse energy have very little effect on the KED. The pulse energy fluctuations are therefore unlikely to cause such a large variance in the kinetic energies.

(2) The X-ray pulses at LCLS are produced by self-amplified spontaneous emission (SASE) and have a spiky structure, rather than the smooth pulse shape indicated by the averaged XTCAV measurement. By performing calculations with randomly sampled pulse spikes, we verified that the spikiness has no effect on the calculated ion yields or on the means and standard deviations of the KED.

(3) The initial conditions in our calculation do not reflect the Wigner distribution of the vibrational ground state of the molecule. Small distortions in the initial molecule geometry due to fluctuations in the vibrational ground state may give rise to a broader KED. To test this idea, we used an instantaneous ionization model, in which the fragments are charged up immediately and the fragmentation happens according to classical propagation of the atoms in the electrostatic field of the charged particles, to estimate the kinetic energy release for various molecular geometries. In the instantaneous model, only variations along the $\mathrm{C}-\mathrm{I}$ stretch mode have meaningful consequences on the kinetic energy release of iodine. By varying the initial C-I bond length (approximately $2.1 \AA$ ) by $\pm 0.04 \AA$-the standard deviation of the Wigner distribution along the $\mathrm{C}-\mathrm{I}$ stretch mode as derived from vibrational mode analysis - we obtain a standard deviation of the KED of less than $2 \%$. The large width observed in the experimental distribution is therefore far beyond this estimated fluctuation caused by the Wigner distribution of the vibrational ground state of the molecule.

(4) For the calculation of electronic transition rates, we computed decay rates and cross-sections for electronic configurations that are an average over multiple states with different spin configurations. However, the decay rates for different spin configurations can vary markedly and so, depending on the spin configuration, faster or slower charging up of the molecular fragments than the average value used in our theoretical model may occur. To test how much this could influence the calculated KED, we performed a calculation in which we varied all of the Auger 


\section{RESEARCH LETTER}

decay rates multiplied by a random, Gaussian-distributed factor with a mean of 1 and a variance of 1 (negative Auger decay rates were set to zero). Using this calculation, we obtained a noticeably broader KED (by about 50\%). From these considerations, we speculate that spin characteristics in the decay cascades are the dominant effect that may lead to a broader KED for the iodine fragments.

Data availability. The data that support the findings of this study are available from the corresponding author upon reasonable request.
31. Boutet, S. \& Williams, G. J. The Coherent X-ray Imaging (CXI) instrument at the Linac Coherent Light Source (LCLS). New J. Phys. 12, 035024 (2010).

32. Murphy, B. F. et al. Femtosecond $\mathrm{X}$-ray-induced explosion of $\mathrm{C}_{60}$ at extreme intensity. Nat. Commun. 5, 4281 (2014).

33. Johnson, R. D. III (ed.) NIST Computational Chemistry Comparison and Benchmark Database, Release 17b. NIST Standard Reference Database Number 101, http://cccbdb.nist.gov/ (National Institute of Standards and Technology, 2015). 


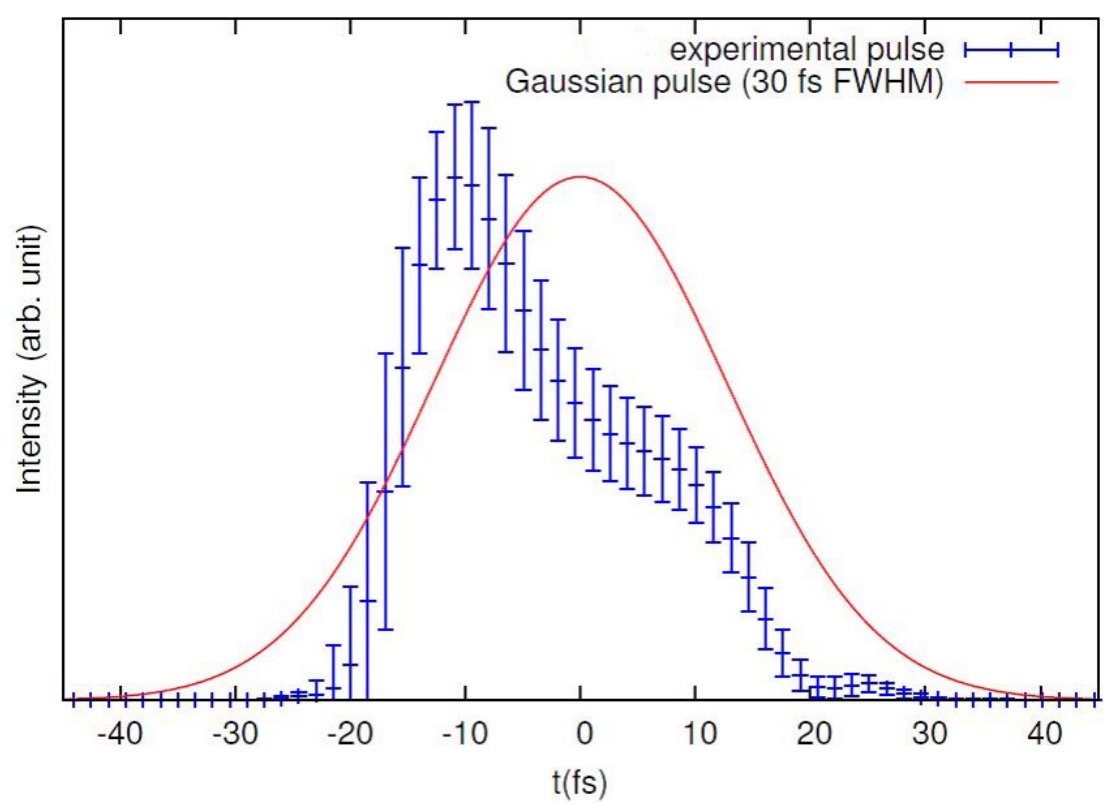

Extended Data Figure 1 | Experimental temporal profile of the X-ray pulse. Averaged experimental X-ray pulse shape (blue), obtained by averaging 369 single-pulse measurements using the X-band transverse deflecting cavity (XTCAV), compared to a Gaussian-shaped model pulse (red). The error bars reflect the standard deviations. 


\section{RESEARCH LETTER}
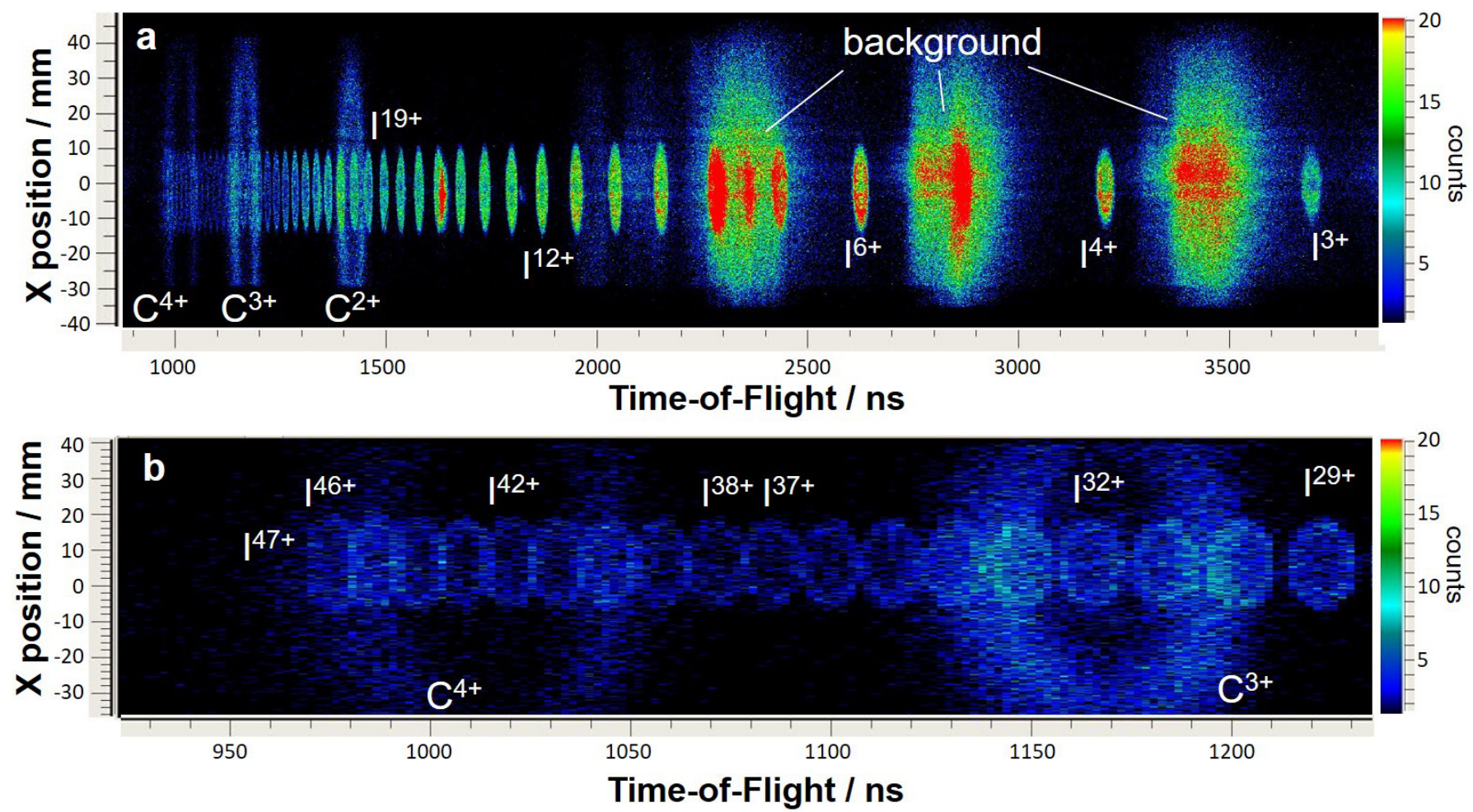

Extended Data Figure 2 Experimental ion yield of $\mathrm{CH}_{3} \mathrm{I}$ after photoionization with ultra-intense LCLS pulses at a photon energy of $8.3 \mathrm{keV}$. a, Ion yield (colour scale) plotted as a function of the measured TOF and hit (X) position on the detector. In addition to the mass-tocharge ratio, this spectrum also shows the scaled momentum distribution of each fragment projected onto the $x-z$ plane, which can be seen as the spread in the measured TOF and position peak for each ion species. The LCLS pulse parameters are the same as for Fig. 1a. The experimental data are accumulated over 287,400 LCLS shots. b, Same as a, but zoomed into the region of short TOFs, that is, high charge states. 


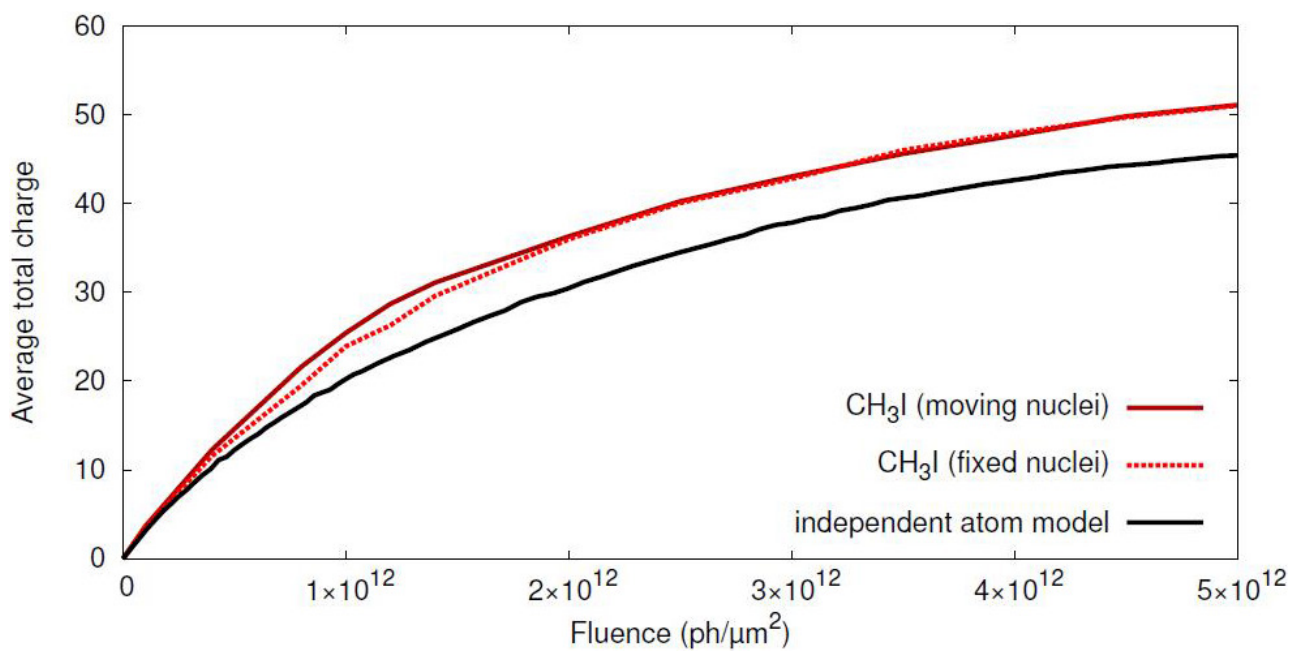

Extended Data Figure 3 Effect of nuclear dynamics on the total

molecular charge. Calculated average total molecular charge of the $\mathrm{CH}_{3} \mathrm{I}$ molecule obtained from the full calculation (solid red line), for fixed nuclear positions (dashed red line), and within the independent atom model (solid black line), each as a function of the X-ray fluence. 


\section{Ion detector}

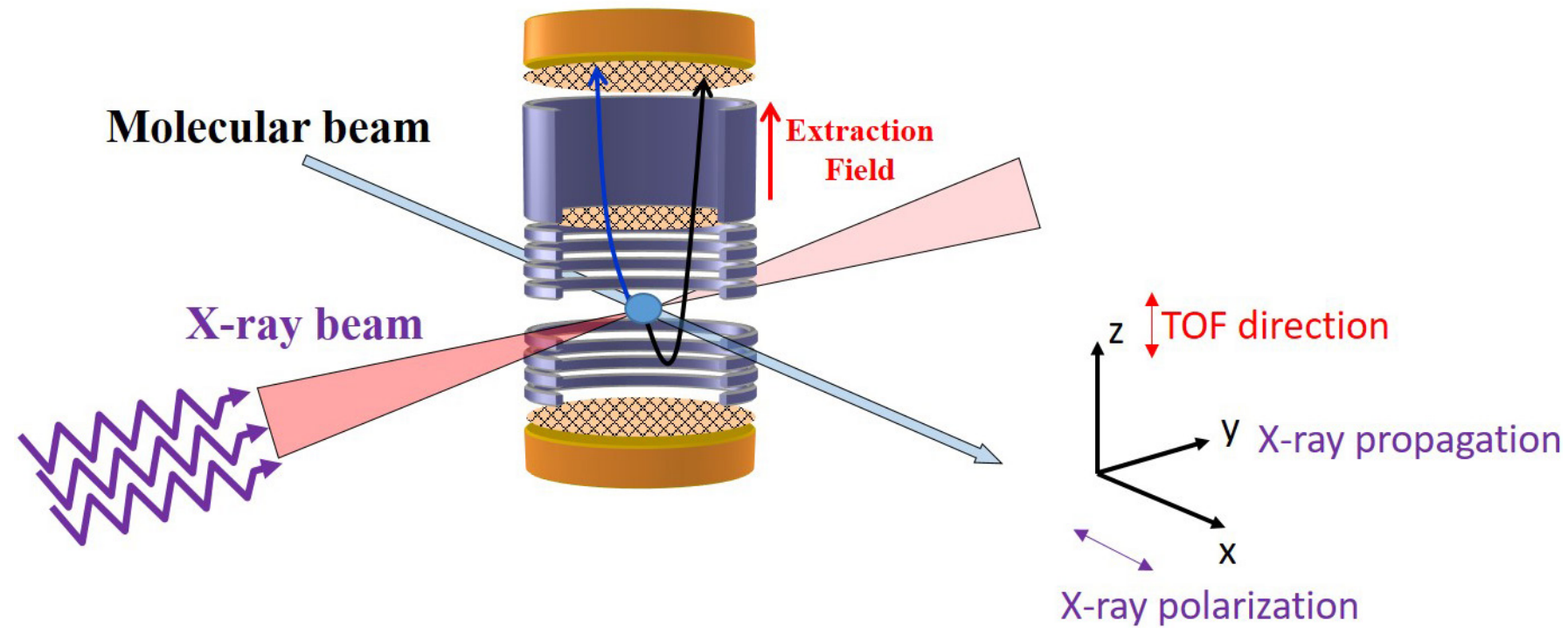

Extended Data Figure $4 \mid$ Sketch of the experimental set-up. The linearly polarized, focused X-ray beam intersects a cold molecular beam inside an ion spectrometer that measures all three components of the ion momentum vectors. Ions are extracted by a constant electric field onto a position- and time-sensitive charged particle detector. From the TOF and hit position of each ion, its three-dimensional momentum vector can be calculated. The size and shape of the X-ray focus was determined from a calibration measurement of the charge-state yield of argon, as described in Methods. 


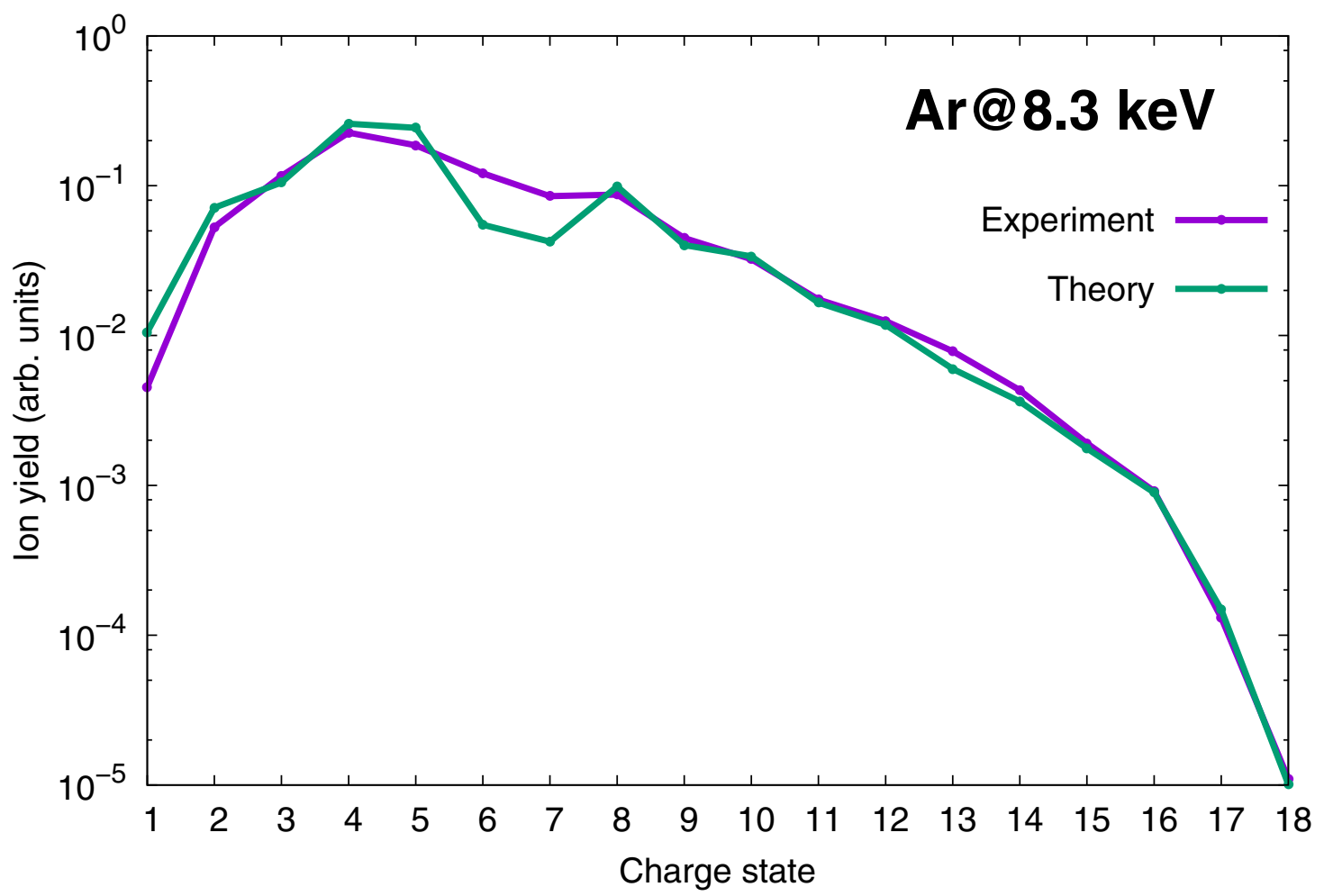

Extended Data Figure 5 | Charge-state distribution of argon. The purple and green lines show the experimental and theoretical charge-state distributions, respectively, of argon atoms at a photon energy of $8.3 \mathrm{keV}$ ('Ar@8.3 keV') and a pulse energy of $3.5 \mathrm{~mJ}$ measured at the gas monitor detector (upstream from beamline optics). The experimental data are accumulated over 52,200 LCLS shots. The calculations assume a

beamline transmission of $32 \%$ and were averaged over a focal spot size of $0.35 \mu \mathrm{m} \times 0.3 \mu \mathrm{m}$ assuming a double-Gaussian spatial distribution. The ratio of peak fluences and widths of the two Gaussians were chosen to be 0.16 and 2.5 , respectively, to best fit the experimental data. 


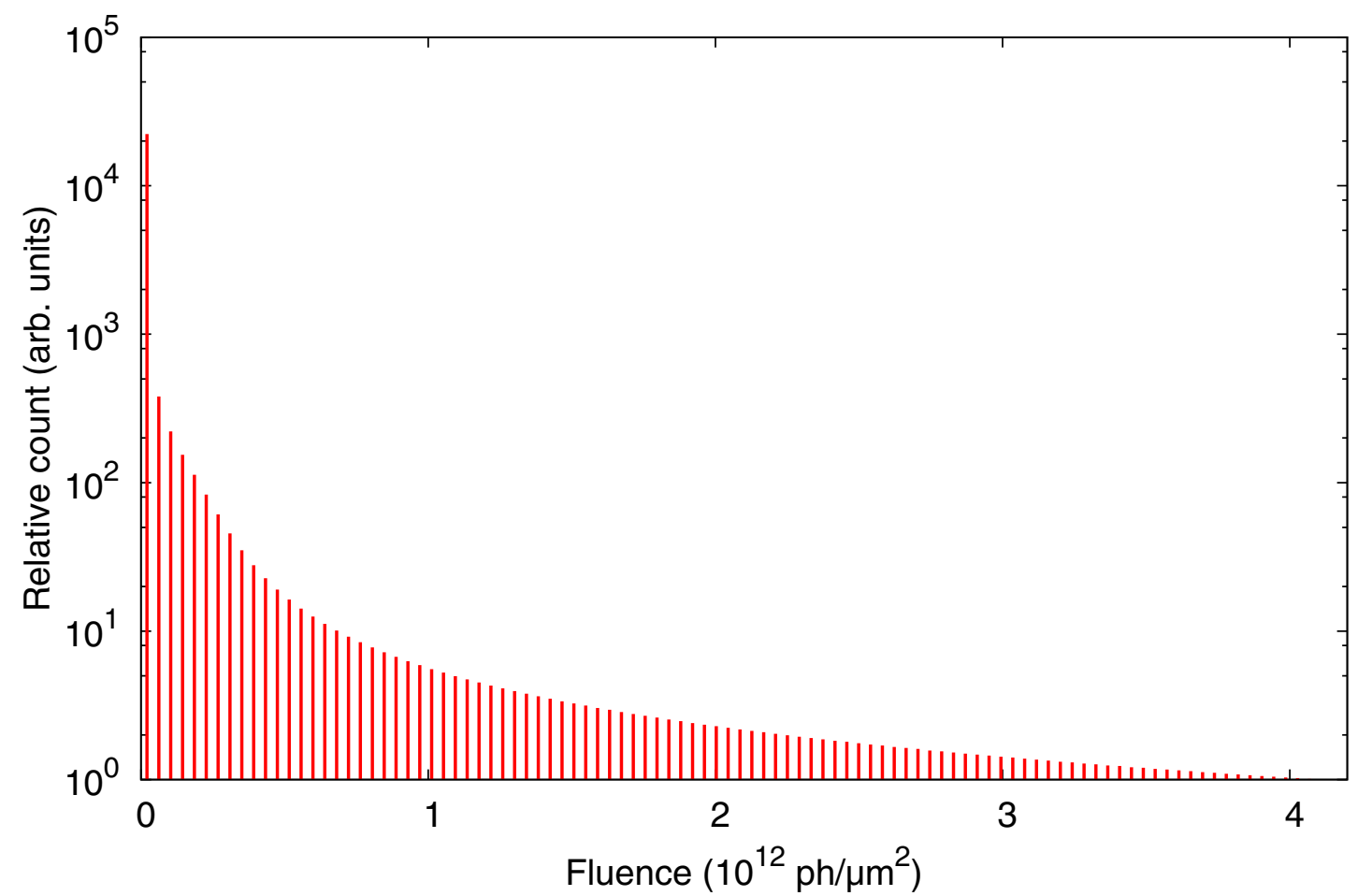

Extended Data Figure 6 | Fluence distribution function obtained from the argon calibration. The peak fluence is $4.13 \times 10^{12}$ photons per $\mu \mathrm{m}^{2}$. The vertical axis shows how often each fluence value occurs in the interaction volume. 


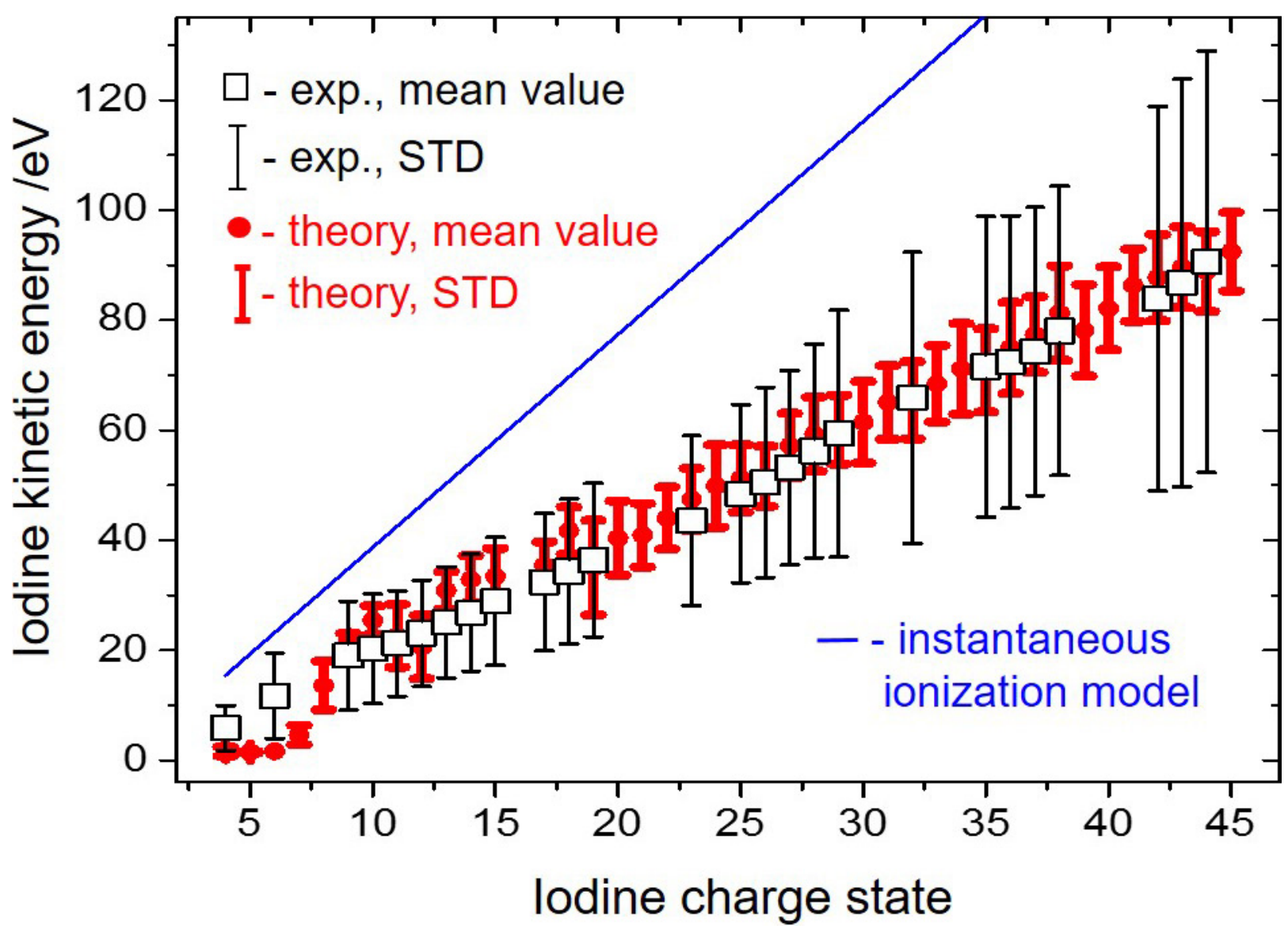

Extended Data Figure 7 | Comparison of the measured and calculated KEDs for $\mathbf{a ~ C H}_{3} \mathrm{I}$ molecule. Means and standard deviations (STDs) of the experimental KEDs of the iodine fragments compared to their calculated values. The LCLS pulse parameters are the same as for

Fig. 1a. The experimental data are accumulated over 287,400 LCLS shots. For the calculation, the experimentally determined X-ray pulse shape shown in Extended Data Fig. 1 was assumed. Note that the vertical bars depict the measured (black) or calculated (red) standard deviations (that is, the width) of the KEDs, not the standard error of the mean as in Fig. 3. The blue line depicts the energies that are expected for instantaneous ionization. 